\title{
THz and microwave properties of 3D-printed nanocarbon based multilayers
}

\author{
P. Kuzhir ${ }^{1}$, A. Paddubskaya ${ }^{1}$, N. Volynets ${ }^{1}$, R. Kotsilkova ${ }^{2}$, E. Ivanov $^{3}$, I. Biró ${ }^{4}$, G. I. \\ Márk $^{5}$, L. P. Biró ${ }^{5,}$ S. Maksimenko ${ }^{1}$
}

\begin{abstract}
We propose a new type of light-weight conductive thin film material having good mechanical properties and electromagnetic shielding efficiency. For that 3D printing through layer-to-layer deposition of nanocarbon containing plastic layers and neat polymer layers was combined with hot pressing to obtain $10 \mu \mathrm{m}$ thick films. We show that such composite has $\operatorname{Re} \varepsilon \approx \operatorname{Im} \varepsilon$ in very broad frequency range, $200 \mathrm{GHz}-0.6 \mathrm{THz}$.
\end{abstract}

\section{INTRODUCTION}

Direct implementation of terahertz ( $\mathrm{THz})$ and subterahertz radiation for applications in home-land security systems, communications and medical imaging, requires materials allowing efficient control and management of electromagnetic (EM) response properties. The absence of a material having simultaneously a high real and imaginary parts of the permittivity in the $\mathrm{THz}$ range limits possibilities in application of passive elements. The idea of the paper is to propose and justify experimentally a novel concept for fabrication of a new generation of materials with high dielectric permittivity and at the same time highly conductive to be suited for $\mathrm{THz}$ and sub-THz ranges. The concept will exploit an idea of $3 \mathrm{D}$ printing of layered structures containing dielectric and conductive planes made of nanocarbon based composite filament, and hot pressing of these structures to achieve outstanding dispersion state. Main emphasis will be given to demonstrate features over a wide range of the electromagnetic (EM) parameters aiming to achieve high dielectric constant material for $\mathrm{THz}$ and microwave applications.

\section{MATERIALS AND STRUCTURES}

We use a commercially available nanocarbon contaning filament Black Magic based on polylactic acid (PLA) thermoplastic doped with graphene nanoplatelets, carbon nanotubes, carbon black, etc. The multilayered structure of initial 3D printed samples was manufactured via layer-to-layer deposition of $100 \mu \mathrm{m}$ PLA layer and $100 \mu \mathrm{m}$ Black Magic layer (BML), repeated as building blocks. The 3D printed samples were then hot pressed at $190^{\circ} \mathrm{C}$, applying a 100 Bar pressure to obtain thin films $\sim 10 \mu \mathrm{m}$. Samples containing 1 to 3 BMLs were fabricated, named BML1, BML2 and BML3.

\section{EXPERIMENTAL}

The microwave measurements in frequency range from $26 \mathrm{GHz}$ to $37 \mathrm{GHz}$ were provided by a Scalar Network Analyzer R2-408R, ELMIKA, Lithuania, at room temperature. The transmitted/input $\left(S_{21}\right)$ and reflected/input $\left(S_{11}\right)$ signals have been measured with frequency stability $10^{-6}$ and power stability $7.0 \mathrm{~mW} \pm$ $10 \mu \mathrm{W}$. Measurement errors of $S_{21}$ were $\delta\left|\mathrm{S}_{21}\right|= \pm(0.6$ $\left.+0.06\left|\mathrm{~S}_{21}\right|\right)$. Reflectance $(\mathrm{R})$, transmittance $(\mathrm{T})$ and absorbance (A) were obtained from measured $S$ parameters as $R=S_{11}^{2}, T=S_{21}^{2}, A=1-R-T$. THz time-domain spectrometer, T-SPEC Ekspla, Lithuania, was used for $\mathrm{THz}$ experiments. Spectrometer is based on femtosecond laser (wavelength $1 \mu \mathrm{m}$, pulse duration less than 150 fs) and GaBiAs photoconductive switch as $\mathrm{THz}$ emitter and detector working at $100 \mathrm{GHz}-3 \mathrm{THz}$.

\section{RESULTS AND DISCUSSION}

One can see that $\operatorname{Re}(\varepsilon)$ and $\operatorname{Im}(\varepsilon)$ decrease with increasing frequency, while their absolute values are close, especially in high frequency range $200-600 \mathrm{GHz}$ $(\tan \delta \sim 1)$, see Figure 1a. The analogous behavior was observed for polymer composites filled with carbon nanotubes [1-3] in case of long tubes for which no EM screening effect of inner walls take place and carbon nanotubes concentration is well above the percolation threshold [4].

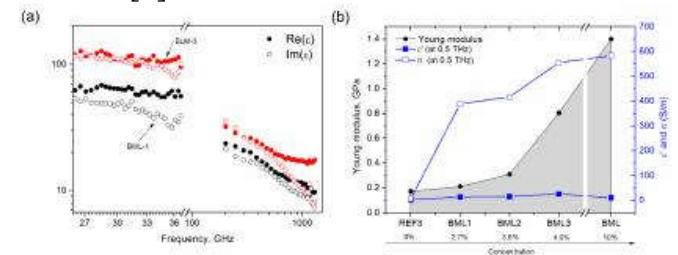

Figure 1: (a) Frequency dependence of $\operatorname{Re}(\varepsilon)$ and Im ( $\varepsilon$ ) for BML1 and BML3 samples. (b) The dependence of the real part of the dielectric permittivity and ac conductivity in the $\mathrm{THz}$ frequency range, and the mechanical properties (black curve) on the number of layers in the original sample (or filler concentration, wt.\%) after hot pressing [5].

Comparative analysis of the mechanical (Young's modulus) and EM properties is shown in Figure 1b vs

\footnotetext{
${ }^{1}$ Institute for Nuclear Problems, Belarusian State University, Minsk, Belarus, e-mail: polina.kuzhirgmail.com

${ }^{2}$ Open Laboratory on Experimental Micro and Nano Mechanics, Institute of Mechanics, Bulgarian Academy of Sciences, Sofia, Bulgaria

${ }^{3}$ NanoTech Lab Ltd., Sofia, Bulgaria

${ }^{4}$ 3D Wishes, Bíró u. Hungary (http://3dkivansag.blog.hu/)

${ }^{5}$ Institute of Technical Physics and Materials Science, Centre for Energy Research, Budapest, Hungary
} 
number of BML layers. An important feature of the composites obtained by hot pressing is the possibility to control precisely the concentration of nanocarbon fillers through the varying of the layers thickness in the initial 3D printed structure and the number of layers. It is important that the maximal possible concentration for hot pressed sample could be $6 \mathrm{wt} . \%$ for "infinite" number of layers in the initial sandwich structure consisting of PLA substrate and equal number of PLA and BML (10 wt.\% of nanocarbon additives) layers.

There are quite a lot of papers devoted to modeling [6-8] and experimental observation $[9,10]$ of dependencies of physical properties of composites vs their microstructure. Many factors have to be taken into account such as (i) functional additives filling fraction, (ii) their functional property, (iii) geometrical parameters (including aspect ratio), (iv) percolation threshold and concentration at which saturation of particular physical properties takes place, (v) issues with composite fabrication (i.e. interaction between filler and matrix), etc. Sometimes it is possible to observe empirically, the simultaneous variation in filler concentration and physical properties [9], or even a particular correlation, that is when various characteristics manifest them self differently with the variation of the concentration according to some correlation law [11]. The case of hot-pressed 3D printed samples is especially interesting because the tendencies of simultaneous improvement of mechanical, electric and EM properties of hot-pressed samples shown in Figure $1 \mathrm{~b}$ for $1 . .3 \mathrm{BML}$ and pure BML layer (made of black magic filament without adding PLA layers and substrate), could be used for prediction of physical behaviors of hot pressed samples depending on the number of layers.

\section{CONCLUSIONS}

Recently it was shown [12] that 3D printed sandwiches made of commercial composite filament Black Magic based on polylactic acid thermoplastic doped with highly conductive nanocarbon fillers may have a great potential for electromagnetic compatibility applications in microwave frequency range, giving EMI SE at the level of $8-15 \mathrm{~dB}$ being 0.4-1 mm thick. Here we shows (see [5] for further details) that further hot pressing gives to the resultant material interesting property: being thin enough in comparison with conventional polymer composites used for EM applications [13], these materials may compete with the CVD graphene sandwiches [14, 15] because of much better processability, and final product cost.

We demonstrate that unlike most conventional composites with pronounced dielectric properties ( $R e \varepsilon$ $>\operatorname{Im} \varepsilon)$ at high frequencies, combination of 3D printing and hot pressing allows to achieve $R e \varepsilon \approx I m$ $\varepsilon$ in very broad frequency range $(0.2-0.6 \mathrm{THz})$. That means much higher absorption ability in comparison with conventional polymer composites together with much smaller thickness (10 microns) that could provide high EMI SE.

\section{Acknowledgments}

The work is supported by EU Project "GRAPHENE Core 1" - n.696656 -FETFLAGSHIP and H2020 RISE 734164 Graphene 3D.

\section{References}

[1] C. Xiang et al. Ceramics International 33, 1293 (2007)

[2] A. Fletcher, M. C. Gupta, K. L. Dudley, and E. Vedeler, Comput. Sci. Technol. 70, 953 (2010)

[3] A. C. Xiang, Y. Pan, X. Liu, X. Sun, X. Shi, and J. Guo, Appl. Phys. Lett. 87, 123103 (2005)

[4] M.V. Shuba, et al, Phys. Rev. B 88, 045436 (8pp) (2013)

[5] R. Kotsilkova, et al, Journal of Applied Physics 121, $064105 \quad$ (2017); doi: http://dx.doi.org/10.1063/1.4975820

[6] D.K. Hale, J Mater Sci, 11, 2105 (1976)

[7] A.P. Roberts and M. A. Knackstedt, Phys. Rev. E 54, 2313 (1996)

[8] S Torquato, International Journal of Solids and Structures, 37 (1-2), 411-422 (2000)

[9] P. Kuzhir, et al, J. Applied Physics, 114 , 164304 (2013)

[10] A. Celzard, et al, Phys.Rev. B 53 (10), 6209 (1996)

[11] B. De Vivo, et al, J. Applied Physics, 116, 054307 (2014)

[12] A. Paddubskaya, et al, J. Appl. Phys., 119, 135102 (2016)

[13] F. Qin, C. Brosseau, J. Appl. Phys., 111, 061301 (2012)

[14] K. Batrakov, et al, Scientific Reports 4, Article number: 7191

[15] K. Batrakov, et al, Appl.Phys. Lett. 108, 123101 (2016) 